\title{
Quantum-noise power spectrum of fields with discrete classical components
}

\author{
Jan Harms, ${ }^{1}$ Paul Cochrane, ${ }^{1}$ and Andreas Freise ${ }^{2}$ \\ ${ }^{1}$ Institut für Gravitationsphysik, Universität Hannover and Max-Planck-Institut für Gravitationsphysik (Albert-Einstein-Institut), Callinstr. \\ 38, 30167 Hannover, Germany \\ ${ }^{2}$ School of Physics and Astronomy, University of Birmingham, Edgbaston, Birmingham B15 2TT, United Kingdom
}

(Received 16 March 2007; published 7 August 2007)

\begin{abstract}
We present an algorithmic approach to calculate the quantum-noise spectral density of photocurrents generated by optical fields with arbitrary discrete classical spectrum in coherent or squeezed states. The measurement scheme may include an arbitrary number of demodulations of the photocurrent. Thereby, our method is applicable to the general heterodyne detection scheme, which is implemented in many experiments. For some of these experiments, e.g., in laser-interferometric gravitational-wave detectors, a reliable prediction of the quantum noise of fields in coherent and squeezed states plays a decisive role in the design phase and detector characterization. Still, our investigation is limited in two ways. First, we consider only coherent and squeezed states of the field, and second, we demand that the photocurrent depends linearly on the field's vacuum amplitudes, which means that at least one of the classical components is comparatively strong.
\end{abstract}

DOI: 10.1103/PhysRevA.76.023803 PACS number(s): 42.50.Dv, 04.80.Nn, 03.65.Ta, 95.55.Ym

\section{INTRODUCTION}

Investigations of the quantum-noise spectrum usually elaborate on the properties and correlations of the quantum vacuum while assuming simple classical components of the field [1-3]. In another publication the authors investigate the quantum-noise contribution to a slightly more complex classical spectrum, i.e., the outcome of a heterodyne power measurement including one subsequent demodulation of the photocurrent [4]. However, the classical spectrum of fields inside real instruments is usually more complex, comprised of many pairs of heterodyning sidebands $[5,6]$. In addition, one may be interested in measurement schemes, which include more than one demodulation of the photocurrent. In this paper, we extend previous analyses by allowing for an arbitrary number of discrete classical components and an arbitrary number of demodulations. Furthermore, our approach is algorithmic, which means that the results can be implemented straightforwardly in the code of simulators of quantum-noise spectra.

In Sec. II, we introduce our notational conventions and calculate spectral densities for power measurements of fields in coherent states. The complexity is gradually increased, starting with a measurement of discrete components without demodulation and ending with multicomponent fields including arbitrarily many demodulations. These results are further generalized in Sec. III to include fields in squeezed states. For this purpose, the representation of the photocurrent is slightly but essentially modified to take account of the intricate sideband correlations occurring in squeezed fields. Finally, in Sec. IV, the algorithm is applied to investigate the photocurrent noise spectrum for a specific multicomponent, squeezed field configuration, which is meant to clarify the abstract approach outlined in previous sections.

\section{QUANTUM-NOISE SPECTRUM OF COHERENT FIELDS}

Let us first calculate the quantum-noise spectral density of a coherent field, which is determined by a single classical amplitude $c_{0}$ at frequency $f_{0}$. Denoting the quantum vacuum noise amplitudes by $\hat{q}(f)$, the electric field can be written

$$
\hat{E}(t)=c_{0} e^{-2 \pi i f_{0} t}+c_{0}^{*} e^{2 \pi i f_{0} t}+\int_{0}^{\infty} d f\left[\hat{q}(f) e^{-2 \pi i f t}+\hat{q}^{\dagger}(f) e^{2 \pi i f t}\right] .
$$

Factoring out the oscillating phase $\exp \left(-2 \pi i f_{0} t\right)$ of the classical field, the quantum vacuum integration is carried out over sideband frequencies $F=f-f_{0}$. The range of sideband frequencies is restricted since we consider a limited measurement bandwidth: $F \in[-B, B]\left(B \ll f_{0}\right)$. We also split the field into two Hermitian conjugate parts $\hat{E}^{(+)}(t), \hat{E}^{(-)}(t)$ and so the positive-frequency field is

$$
\hat{E}^{(+)}(t)=e^{-2 \pi i f_{0} t}\left(c_{0}+\int_{-B}^{B} d F \hat{q}\left(f_{0}+F\right) e^{-2 \pi i F t}\right) .
$$

When talking of spectral densities, we mean the power spectral density of the photocurrent after demodulations. Ideally, the photocurrent $\hat{I}(t)$ is proportional to the power of the field. For real photodiodes, this does not have to be true for arbitrarily large detection bandwidths. In the simplest case the current spectral density has to be multiplied with a factor, which accounts for the frequency dependent response of the photodiode. Let us assume that $B$ is small enough so that $\hat{I}(t) \propto \hat{P}(t)$. The power of the field Eq. (2) averaged over a period much smaller than $c / B$ is given by

$\hat{P}(t)=\hat{E}^{(-)}(t) \hat{E}^{(+)}(t)=\frac{1}{2}\left|c_{0}\right|^{2}+\int_{-B}^{B} d F c_{0}^{*} \hat{q}\left(f_{0}+F\right) e^{-2 \pi i F t}+$ H.c.

Contributions quadratic in the vacuum amplitudes are neglected, which is a valid approximation whenever $P_{0} \equiv\left|c_{0}\right|^{2}$ $\gg h f_{0} / \tau$, where $\tau$ denotes the measurement time. It follows that the optical quantum noise of the photocurrent $\hat{I}^{\mathrm{QM}}(t)$ is proportional to 


$$
\hat{I}^{\mathrm{QM}}(t) \propto \int_{-B}^{B} d F c_{0}^{*} \hat{q}\left(f_{0}+F\right) e^{-2 \pi i F t}+\text { H.c. }
$$

The proportionality factor $\chi$ between current and light power-the photodiode responsivity-typically assumes values $\chi=0.2-0.7 \mathrm{~A} / \mathrm{W}$ at $f \sim 3 \times 10^{14} \mathrm{~Hz}$ [7]. Since subsequent calculations are based on a frequency independent responsivity, there is no real distinction between light power and photocurrent power spectral densities except for a factor of $\chi^{2}$. Throughout this paper the explicit dependence of the photocurrent on $\chi$ is omitted.

Vacuum amplitudes like any classical amplitudes of stationary noise are numerically ill defined (the Fourier transform of stationary noise being infinite) and serve exclusively as algebraically meaningful quantities. Nevertheless, the power spectral density of stationary noise is well defined and there exists a simple relationship between stationary vacuum noise amplitudes $\hat{q}$ and its (single-sided) noise spectral densities $S_{q}$,

$$
\frac{1}{2} \delta\left(f-f^{\prime}\right) S_{q}(f)=\frac{1}{2}\left\langle\hat{q}(f) \hat{q}^{\dagger}\left(f^{\prime}\right)+\hat{q}^{\dagger}\left(f^{\prime}\right) \hat{q}(f)\right\rangle=\frac{1}{2} \delta\left(f-f^{\prime}\right) h f .
$$

The corresponding quantum noise spectral density of the photocurrent Eq. (4), which results from the power measurement of a coherent field reads [8]

$$
S_{\mathrm{I}}^{\mathrm{QM}}(F)=\left|c_{0}\right|^{2} S_{q}\left(f_{0}\right)=P_{0} h f_{0} .
$$

The invalidity of the latter equation for squeezed states of the field and the fact that the spectral density does not depend on the sideband frequency $F$ is clarified in Sec. III [see Eq. (28)]. At this point, we only wish to draw attention to the apparently simple algorithm for coherent fields, which leads to Eq. (6) starting from Eq. (2): square the classical amplitude and multiply the result by $h f_{0}$.

In fact, the algorithm stays valid for a wider class of power measurements. Consider a set of classical components with amplitudes $c_{\mathrm{i}} \equiv c\left(f_{\mathrm{i}}\right), i \in\{0,1, \ldots, N-1\}$. Like before, we factor out the phase of the component $c_{0}$, which formally converts all optical oscillations into sideband oscillations with respect to the reference frequency $f_{0}$. Defining $F_{\mathrm{i}} \equiv f_{i}$ $-f_{0}$, the positive-frequency field is cast into the form

$$
\hat{E}^{(+)}(t)=e^{-2 \pi i f_{0} t} \sum_{i=0}^{N-1}\left(c_{i} e^{-2 \pi i F_{i} t}+\int_{F_{i}-B}^{F_{i}+B} d F \hat{q}\left(f_{0}+F\right) e^{-2 \pi i F t}\right) .
$$

We demand that $P_{i} \equiv\left|c_{i}\right|^{2} \gg h f_{0} / \tau$ is valid for all classical components. If that condition did not hold for some classical components (typically for signal sidebands), then the respective amplitudes would not influence the quantum-noise power spectrum as long as at least one high power component exists and so for our purpose we may safely neglect weak components. A power measurement yields a photocurrent whose quantum noise is determined by

$$
\begin{aligned}
\hat{I}^{\mathrm{QM}}(t) & =\sum_{i=0}^{N-1} \int_{F_{i}-B}^{F_{i}+B} d F c_{i}^{*} \hat{q}\left(f_{0}+F\right) e^{-2 \pi i\left(F-F_{i}\right) t}+\text { H.c. } \\
& =\sum_{i=0}^{N-1} \int_{-B}^{B} d F c_{i}^{*} \hat{q}\left(f_{0}+F+F_{i}\right) e^{-2 \pi i F t}+\text { H.c. }
\end{aligned}
$$

Provided that all intervals $\left[F_{i}-B, F_{i}+B\right]$ are mutually disjointed, the quantum-noise power spectral density of the photocurrent is given by

$$
S_{\mathrm{I}}^{\mathrm{QM}}(F)=\sum_{i=0}^{N-1}\left|c_{i}\right|^{2} S_{q}\left(f_{0}+F_{i}\right)=\sum_{i=0}^{N-1} h\left(f_{0}+F_{i}\right) P_{i} .
$$

Unfortunately, there are no more simple example cases that could be presented. Let us now skip to the next section and introduce demodulations of photocurrents.

\section{A. Single demodulation}

There are two well-known demodulation techniques. Either the photocurrent is multiplied by a harmonic function $\hat{I}(t) \cos (2 \pi D t+\phi)$ or one rectifies the photocurrent, which means the final output is $|\hat{I}(t)|$. In practice, the low-frequency spectra $\left(B \ll F_{i}\right)$ drawn from these two outputs differ by a constant factor, the spectrum of the harmonic demodulation being smaller by a factor of $(\pi / 4)^{2}$. Anyway, the spectrum of the harmonically demodulated current is calculable by much simpler algebra. In addition, we want to study measurement schemes, which implement multiple demodulations characterized by a set of demodulation frequencies $D_{i}$ and demodulation phases $\phi_{i}$. For these two reasons, we consider harmonic demodulations throughout this paper. Let us perform a single demodulation of the current Eq. (8),

$$
\begin{aligned}
\hat{I}^{\mathrm{QM}}(t)= & \frac{1}{2} \sum_{i=0}^{N-1} \int_{-D-B}^{-D+B} d F c_{i}^{*} \hat{q}\left(f_{0}+F+F_{i}\right) e^{-i[2 \pi(F+D) t+\phi]} \\
& +\frac{1}{2} \sum_{i=0}^{N-1} \int_{D-B}^{D+B} d F c_{i}^{*} \hat{q}\left(f_{0}+F+F_{i}\right) e^{-i[2 \pi(F-D) t-\phi]} \\
& + \text { H.c. }
\end{aligned}
$$

or shifting the frequency range, the current noise finally assumes the form

$$
\begin{aligned}
\hat{I}^{\mathrm{QM}}(t)= & \frac{1}{2} \sum_{i=0}^{N-1} \int_{-B}^{B} d F e^{-2 \pi i F t}\left[c_{i}^{*} e^{-i \phi} \hat{q}\left(f_{0}+F+F_{i}-D\right)\right. \\
& \left.+c_{i}^{*} e^{i \phi} \hat{q}\left(f_{0}+F+F_{i}+D\right)\right]+ \text { H.c. }
\end{aligned}
$$

Now, if all frequency intervals $\left[F_{i}-D-B, F_{i}-D+B\right],\left[F_{i}\right.$ $\left.+D-B, F_{i}+D+B\right]$ are mutually disjointed, then one obtains the same spectral density as in Eq. (9) except for an additional factor $1 / 2$, which is basically due to the fact that by demodulating we multiply the photocurrent with a function which, in terms of power, has a gain of $1 / 2$. However, this situation is uncommon in real experiments.

It is time to introduce a graphical auxiliary in order to understand what is happening. For simplicity we start with 


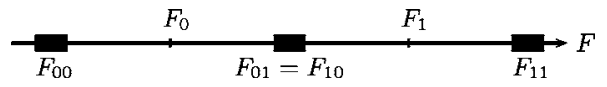

FIG. 1. Representation of the relevant frequency ranges for a classical heterodyne power measurement. The black boxes indicate the detection bandwidth.

two classical components-a carrier and a subcarrier-at frequencies $F_{0}=0 \mathrm{~Hz}$ and $F_{1}=30 \mathrm{MHz}$. The photocurrent is demodulated with $D=15 \mathrm{MHz}$ and the detection bandwidth is limited to $B=1000 \mathrm{~Hz}$. This choice of frequencies also corresponds to a very common situation in optical experiments, namely, the heterodyne measurement where the two classical components are generated by a $15 \mathrm{MHz}$ modulation of a carrier field, which oscillates at high optical frequencies $\left(f_{0}\right.$ $\sim 10^{15} \mathrm{~Hz}$ ). A discussion of this problem focusing on signalto-noise ratios can be found in [4]. We point out that $B$ $\ll\left|F_{0} \pm D\right|,\left|F_{1} \pm D\right|,\left|F_{0} \pm F_{1}\right|$, which significantly simplifies the problem. The four frequency values $F_{00} \equiv F_{0}-D, F_{01}$ $\equiv F_{0}+D, F_{10} \equiv F_{1}-D$, and $F_{11} \equiv F_{1}+D$ are marked on a frequency axis (see Fig. 1) and collected inside a matrix $\mathcal{F}(N$ $=2,2)=\left\{F_{00}, F_{01} ; F_{10}, F_{11}\right\}$. Since two intervals identically overlap, each vacuum amplitude at frequencies inside that interval enters twice into the spectral density calculation. These two contributions have to be added coherently before taking the absolute square. The expression Eq. (11) for the current tells us that all vacuum amplitudes $\hat{q}\left(f_{0}+F\right.$ $+15 \mathrm{MHz}$ ) have to be added coherently. In conclusion, the spectral density in this particular example turns out to be

$$
\begin{aligned}
S_{I}^{\mathrm{QM}}(F)= & \frac{1}{4}\left[h\left(f_{0}-15 \mathrm{MHz}\right) P_{0}+h\left(f_{0}+15 \mathrm{MHz}\right) \mid c_{0}^{*} e^{-i \phi}\right. \\
& \left.+c_{1}^{*} e^{\left.i \phi\right|^{2}}+h\left(f_{0}+45 \mathrm{MHz}\right) P_{1}\right] \\
\approx & \frac{h f_{0}}{4}\left(P_{0}+P_{1}+\mid c_{0}^{*} e^{-i \phi}+c_{1}^{*} e^{i \phi \mid}\right) .
\end{aligned}
$$

Henceforth, we will always assume that overlapping frequency ranges represented by black boxes in Fig. 1 overlap completely, but never partially. The overlap condition guarantees that whenever two frequencies inside the frequency matrix $\mathcal{F}$ do not coincide, then the two respective detection ranges do not overlap and one does not have to worry about coherent summation of the respective amplitudes. This is certainly a reasonable demand for a first approach to an algorithmic realization of the calculation.

We are now prepared to calculate the spectral density for measurements with a single demodulation and an arbitrary number of classical components. It is not possible to give explicit results, because as we have seen these depend on the chosen set of field and demodulation frequencies. Our focus lies on extending the algorithm, which leads to the spectral density. Consider $N$ classical components $c_{i}$ at frequencies $F_{\mathrm{i}}$ and a single demodulation of the photocurrent. The first step is to calculate the frequency matrix

$$
\mathcal{F}(N, 2)=\left(\begin{array}{cc}
F_{00} & F_{01} \\
F_{10} & F_{11} \\
\vdots & \vdots \\
F_{N-1,0} & F_{N-1,1}
\end{array}\right),
$$

where $F_{i 0} \equiv F_{i}-D$ and $F_{i 1} \equiv F_{i}+D$. The second step is to find coinciding frequencies of the matrix. Use the matrix indices of these pairs, e.g., $\left(n_{1}, d_{1}\right)$ and $\left(n_{2}, d_{2}\right)$, to calculate the contribution to the spectral density as follows

$$
\begin{aligned}
S_{\mathrm{I}}^{\mathrm{QM}}\left(F,\left(n_{1}, d_{1}\right),\left(n_{2}, d_{2}\right)\right)= & \frac{h\left(f_{0}+F_{n_{1} d_{1}}\right)}{4} \mid c_{n_{1}}^{*} e^{(-1)^{d_{1}+1} i \phi} \\
& +\left.c_{n_{2}}^{*} e^{(-1)^{d_{2}+1} i \phi}\right|^{2} .
\end{aligned}
$$

All remaining unique frequencies $F_{n d}$ contribute according to

$$
S_{\mathrm{I}}^{\mathrm{QM}}(F,(n, d))=\frac{h\left(f_{0}+F_{n d}\right)}{4}\left|c_{n}\right|^{2} .
$$

In most experiments $f_{0} \gg F_{n d}$ and the quantum vacuum energies can be approximated by $h f_{0}$. Finally, one has to sum up all these contributions.

Let us summarize our preliminary results as a list to be processed when calculating the current noise spectral density:

(1) Calculate the matrix $\mathcal{F}$ according to Eq. (13).

(2) Collect index pairs $(n, d)$ of coinciding frequencies $F_{\text {nd }}$ inside $\mathcal{F}$.

(3) Collect contributions to the current noise spectral density from unique frequencies, which are determined by Eq. (15).

(4) Collect contributions to the current noise spectral density from coinciding frequencies, which are determined by Eq. (14).

(5) Sum up all contributions.

This algorithm needs an extension in order to account for multiple demodulations. This task is accomplished in the next section.

\section{B. $M$ demodulations}

Methodically, increasing the number of demodulations means to increase the number or range of indices in the previous calculations. The first step of the algorithm is always to collect all relevant frequencies inside a matrix. Again, we consider a set of $N$ classical components $c_{i}$ at frequencies $F_{i}$. The demodulations are determined by $M$ demodulation frequencies $D_{i}$ and phases $\phi_{i}$. The final output of the measurement is given by

$$
\begin{aligned}
\hat{I}(t) \prod_{i=0}^{M-1} \cos \left(2 \pi D_{i} t+\phi_{i}\right)= & \hat{I}(t) \frac{1}{2^{M}} \sum_{i=1}^{2^{M}} \cos \left(\sum_{j=0}^{M-1}(-1)^{\left\lfloor i / 2^{M-j-1}\right\rfloor}\right. \\
& \left.\times\left(2 \pi D_{j} t+\phi_{j}\right)\right)
\end{aligned}
$$

with $\lfloor x\rfloor$ denoting the decimal truncating floor function. Evaluating Eq. (16) for a few small number of demodula- 


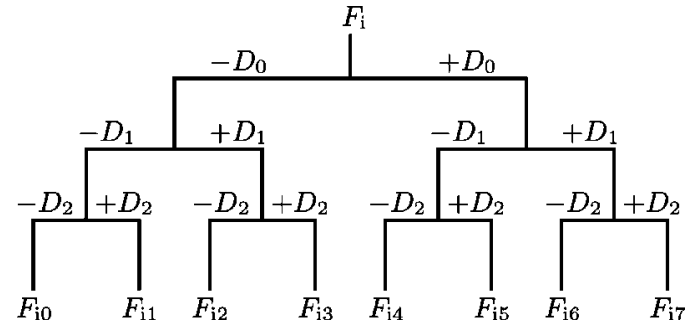

FIG. 2. Sorting scheme for three demodulation frequencies.

tions, one recognizes that the arguments of the harmonic functions consist of all possible \pm combinations of modulation frequencies and phases. Since the complete sum of these harmonic functions is multiplied with the photocurrent, it should be obvious that each row $i$ of the frequency matrix $\mathcal{F}$ contains all frequencies of the form $F_{\mathrm{i}} \pm D_{0} \pm \cdots \pm D_{M-1}$. In which order should these $2^{M}$ frequencies appear? One may argue that the order does not matter, in principle. However, a specific combination of frequencies is associated with an analogous combination of demodulation phases, which then appears in formulas such as Eq. (14). If two frequencies of $\mathcal{F}$ turn out to be equal, then the two pairs of matrix indices have to encode the \pm combination, which determines the respective combination of phases. Algorithmic tractability of the problem requires a good sorting scheme of these frequencies. We propose a sorting scheme, which is derived from a tree structure like that in Fig. 2. The frequencies themselves do not obey $F_{i 0}<F_{i 1}<\cdots$ since we do not assume a magnitudesorted vector of demodulation frequencies. Now, the remaining problem is to derive from each column index the respective combination of demodulation phases. If each row vector of $\mathcal{F}$ is tree sorted then one first converts the decimal column index $j$ into a binary number $(j)_{\text {bin }}=\left[b_{0} b_{1} b_{2} b_{3} \cdots b_{M-1}\right]$ and then calculates a total phase according to

$$
\phi(j)=-\sum_{i=0}^{M-1}(-1)^{b_{i}} \phi_{i}
$$

This phase is to be used in order to calculate contributions to the quantum-noise spectral density when $C$ frequencies of $\mathcal{F}$ coincide. If the respective pairs of indices are $\left(n_{j}, d_{j}\right), j$ $\in\{0, \ldots, C-1\}$, then the spectral density reads

$$
S_{\mathrm{I}}^{\mathrm{QM}}\left(F,\left\{\left(n_{j}, d_{j}\right)\right\}\right)=\frac{h\left(f_{0}+F_{n_{0} d_{0}}\right)}{4^{M}}\left|\sum_{j=0}^{C-1} c_{n_{j}}^{*} e^{i \phi\left(d_{j}\right)}\right|^{2} .
$$

The contribution from unique frequencies $F_{n d}$ is given by

$$
S_{\mathrm{I}}^{\mathrm{QM}}(F,(n, d))=\frac{h\left(f_{0}+F_{n d}\right)}{4^{M}}\left|c_{n}\right|^{2} .
$$

Finally, let us apply the algorithm to the case with $N=2$ classical components and $M=3$ demodulations. The respective frequency matrix is written

$$
\mathcal{F}(2,8)=\left(\begin{array}{llllllll}
F_{00} & F_{01} & F_{02} & F_{03} & F_{04} & F_{05} & F_{06} & F_{07} \\
F_{10} & F_{11} & F_{12} & F_{13} & F_{14} & F_{15} & F_{16} & F_{17}
\end{array}\right) .
$$

Coincidences are found say between frequencies $F_{05}$ and $F_{11}$. The binary representation of the first column index is $(5)_{\text {bin }}$ $=101$ and $(1)_{\mathrm{bin}}=001$ for the second. According to Eq. (17), one obtains $\phi(5)=\phi_{0}-\phi_{1}+\phi_{2}$ and $\phi(1)=-\phi_{0}-\phi_{1}+\phi_{2}$. The respective spectral density is given by

$$
S_{\mathrm{I}}^{\mathrm{QM}}(F,(0,5),(1,1))=\frac{h\left(f_{0}+F_{11}\right)}{64}\left|c_{0}^{*} e^{i \phi(5)}+c_{1}^{*} e^{i \phi(1)}\right|^{2},
$$

which does not depend on $\phi_{1}$ and $\phi_{2}$. Contributions coming from unique frequencies are calculated as usual. The first part of this paper is finished. We have treated the calculation of quantum noise when the field is coherent, which entails that the noise is time stationary. In fact, the algorithm is valid for any type of time-stationary noise including technical laser noise when phase and amplitude noise are not correlated. In that case, one has to substitute the quantum vacuum energies in Eqs. (18) and (19) by another noise spectral density, which characterizes the power of the technical noise at the photodiode. The next step is to take into account the intricate correlation between quantum-noise amplitudes at different frequencies due to squeezing, or in other words, due to amplitude-phase correlations.

\section{QUANTUM-NOISE SPECTRUM OF SQUEEZED FIELDS}

A widely applied mechanism, which correlates vacuum noise amplitudes at different frequencies is the generation of squeezed fields. Squeezed fields are formed in nonlinear crystals [9] and theory predicts that ponderomotive squeezing occurs when light is reflected from suspended mirrors [10]. In order to understand the meaning of squeezing, one has to know that correlations are built up between sideband frequencies $F$ with respect to a reference frequency $f_{\mathrm{i}}$. The squeezing transformation of fields is characterized by a squeezing factor $r$, which quantifies the strength of correlations between different frequencies and a squeezing phase $\phi$. Perfect correlation corresponds to a squeezing factor $r=\infty$. In practice, the reference frequency is realized by means of a single classical component, which is named seed or carrier field depending on whether the squeezing is generated by crystals or ponderomotively. Now, if $\phi=0$ one can show that the correlation between sidebands diminishes the quantum amplitude noise of the classical field and for $\phi=\pi / 2$, the quantum phase noise is decreased. Interpretation of the squeezing phase for intermediate values requires a more sophisticated representation of fields [8]. In principle, the reference frequency does not have to be related to a classical 
field. In that case, the squeezing phase has no ad hoc interpretation. Denoting amplitudes of squeezed fields by $\hat{s}$ and amplitudes of coherent fields by $\hat{q}$, the squeezing transformation is governed by [8]

$$
\hat{s}\left(f_{\mathrm{i}} \pm F\right)=\cosh (r) \hat{q}\left(f_{i} \pm F\right)+\sinh (r) e^{2 i \phi} \hat{q}^{\dagger}\left(f_{i} \mp F\right) .
$$

How does this squeezing transformation relate to our previous investigation of quantum-noise spectral densities? To find an answer, we have to return to Eq. (8). That equation determines the time-dependent photocurrent where $F$, the former optical sideband frequencies, now become true frequencies of the current spectrum. Similarly to expansions of electric fields, we should conform to a strict decomposition into positive and negative frequencies

$$
\begin{aligned}
\hat{I}^{\mathrm{QM}}(t)= & \sum_{i=0}^{N-1} \int_{0}^{B} d F e^{-2 \pi i F t}\left[c_{i}^{*} \hat{s}\left(f_{0}+F+F_{i}\right)\right. \\
& \left.+c_{i} \hat{S}^{\dagger}\left(f_{0}-F+F_{i}\right)\right]+ \text { H.c. },
\end{aligned}
$$

where the vacuum amplitudes have been renamed to indicate the possibility of squeezing. Thereby, the impact of Eq. (22) on the power spectral density of the photocurrent becomes clear. Two amplitudes at different frequencies of the optical vacuum are added to form a single amplitude of the photocurrent. If these two amplitudes are uncorrelated then the spectral density is calculated as before making implicit use of the identity

$$
S\left(\hat{q}_{1}+\hat{q}_{2}\right)=S\left(\hat{q}_{1}\right)+S\left(\hat{q}_{2}\right), \quad \hat{q}_{1}, \hat{q}_{2} \text { uncorrelated. }
$$

The optical field may exhibit correlations due to squeezing and the latter equation can no longer be applied. So the remaining problem is the calculation of the modified spectral density depending on the squeezing factor and phase. There exist two possibilities. Either squeezing is generated whose reference frequency coincides with one of the frequencies $f_{0}+F_{i}$, which appear in the current expansion Eq. (23) and the squeezing factor is significant only within a frequency range comparable to the detection bandwidth $B$, or the squeezing violates either of these two conditions. We start with an investigation of the case when both conditions are fulfilled, which is in some sense the only expedient one. It is then also straightforward to treat fields, which are squeezed at some or all of the frequencies $f_{0}+F_{i}$, with potentially different factors and phases. The correction is derived from the quantum-noise amplitude of the photocurrent;

$$
\hat{p}\left(F, c_{i}^{*}, F_{i}\right) \equiv c_{i}^{*} \hat{s}\left(f_{0}+F+F_{i}\right)+c_{i} \hat{s}^{\dagger}\left(f_{0}-F+F_{i}\right) .
$$

Before or after squeezing, the field may be subject to linear (frequency preserving) transformations such as propagations or reflections from fixed mirrors. If some mirrors are suspended then already squeezed fields experience further squeezing. Consequently, a more generic case is considered here described by the following pair of transformations:

$$
\hat{s}\left(f_{i}+F\right)=t_{00}\left(f_{i}+F\right) \hat{q}\left(f_{i}+F\right)+t_{01}\left(f_{i}-F\right) \hat{q}^{\dagger}\left(f_{i}-F\right),
$$

$$
\hat{s}^{\dagger}\left(f_{i}-F\right)=t_{10}\left(f_{i}+F\right) \hat{q}\left(f_{i}+F\right)+t_{11}\left(f_{i}-F\right) \hat{q}^{\dagger}\left(f_{i}-F\right) .
$$

The two transfer functions $t_{10}, t_{01}$ map input amplitudes to output amplitudes at the photodiode with mutually different frequencies, whereas $t_{00}, t_{11}$ comprise nonlinear and linear transfers as mentioned above. The input vacuum is coherent and therefore all amplitudes $\hat{q}$ of the input vacuum are uncorrelated. Inserting the last equation into the amplitude of the photocurrent and defining $t_{m n}^{i}( \pm) \equiv t_{m n}\left(f_{0} \pm F+F_{i}\right)$, one obtains

$$
\begin{aligned}
\hat{p}\left(F, c_{i}^{*}, F_{i}\right)= & {\left[c_{i}^{*} t_{00}^{i}(+)+c_{i} t_{10}^{i}(+)\right] \hat{q}\left(f_{0}+F+F_{i}\right) } \\
& +\left[c_{i}^{*} t_{01}^{i}(-)+c_{i} t_{11}^{i}(-)\right] \hat{q}^{\dagger}\left(f_{0}-F+F_{i}\right) .
\end{aligned}
$$

The power spectral density associated with the noise amplitude $\hat{p}\left(F, c_{i}^{*}, F_{i}\right)$ is given by

$$
\begin{aligned}
S\left(\hat{p}\left(F, c_{i}^{*}, F_{i}\right)\right)= & \frac{h\left(f_{0}+F_{i}\right)}{2}\left[\mid c_{i}^{*} t_{00}^{i}(+)\right. \\
& +\left.c_{i} t_{10}^{i}(+)\right|^{2}\left(1+\frac{F}{f_{0}+F_{i}}\right)+\mid c_{i}^{*} t_{01}^{i}(-) \\
& \left.+\left.c_{i} t_{11}^{i}(-)\right|^{2}\left(1-\frac{F}{f_{0}+F_{i}}\right)\right] .
\end{aligned}
$$

First, this equation explains the observation that the spectral density of photocurrents when measuring coherent fields is white (i.e., frequency independent). For coherent fields $\left(t_{01}\right.$ $\left.=t_{10}=0,\left|t_{00}\right|^{2}=\left|t_{11}\right|^{2}=1\right)$, the two absolute squares can be substituted by $\left|c_{i}\right|^{2}$ and the dependence on $F$ cancels. Since in most practical situations $f_{0} \gg F$, it is also common to omit the frequency dependence of the spectrum for squeezed states.

The next step is to include demodulations of the photocurrent. In terms of the amplitudes defined in Eq. (27), a singly demodulated photocurrent, Eq. (11), assumes the form

$$
\begin{aligned}
\hat{I}^{\mathrm{QM}}(t)= & \frac{1}{2} \sum_{i=0}^{N-1} \int_{0}^{B} d F e^{-2 \pi i F t}\left[\hat{p}\left(F, c_{i}^{*} e^{-i \phi}, F_{i}-D\right)\right. \\
& \left.+\hat{p}\left(F, c_{i}^{*} e^{i \phi}, F_{i}+D\right)\right]+ \text { H.c. }
\end{aligned}
$$

Hereupon, a simple analogy argument leads to the fullfledged multiple demodulation power spectral density of the photocurrent including squeezed states of the field. If $C$ frequencies with indices $\left(n_{j}, d_{j}\right), j \in\{0, \ldots, C-1\}$ of the frequency matrix $\mathcal{F}$ coincide, then the respective contribution to the power spectral density is determined by [compare with Eq. (18)] 


$$
\begin{aligned}
S_{I}^{\mathrm{QM}}\left(F,\left\{\left(n_{j}, d_{j}\right)\right\}\right)= & \frac{1}{4^{M}} S\left(\sum_{j=0}^{C-1} \hat{p}\left(F, c_{n_{j}}^{*} e^{i \phi\left(d_{j}\right)}, F_{n_{0} d_{0}}\right)\right)=\frac{h\left(f_{0}+F_{n_{0} d_{0}}\right)}{2 \times 4^{M}}\left[\mid \sum_{j=0}^{C-1}\left[c_{n_{j}}^{*} e^{i \phi\left(d_{j}\right)} t_{00}\left(f_{0}+F+F_{n_{0} d_{0}}\right)+c_{n_{j}} e^{-i \phi\left(d_{j}\right)} t_{10}\left(f_{0}+F\right.\right.\right. \\
& \left.\left.+F_{n_{0} d_{0}}\right)\right]\left.\right|^{2}\left(1+\frac{F}{f_{0}+F_{n_{0} d_{0}}}\right)+\left|\sum_{j=0}^{C-1}\left[c_{n_{j}}^{*} e^{i \phi\left(d_{j}\right)} t_{01}\left(f_{0}-F+F_{n_{0} d_{0}}\right)+c_{n_{j}} e^{-i \phi\left(d_{j}\right)} t_{11}\left(f_{0}-F+F_{n_{0} d_{0}}\right)\right]\right|^{2} \\
& \left.\times\left(1-\frac{F}{f_{0}+F_{n_{0} d_{0}}}\right)\right],
\end{aligned}
$$

and unique frequencies $F_{n d}$ contribute with

$$
S_{I}^{\mathrm{QM}}(F,(n, d))=\frac{1}{4^{M}} S\left(\hat{p}\left(F, c_{n}, F_{n d}\right)\right) .
$$

The spectral density on the right-hand side is determined by Eq. (28).

We conclude this section with a brief discussion of "offcentered" squeezing, i.e., some of the squeezing reference frequencies of the field do not coincide with any of the frequencies $F_{n d}$ or that squeezing factors $r$ decay over frequency ranges, which are comparable to $F_{n d} \gg B$. We leave a detailed investigation of both problems for the future. One reason is that in each case amplitudes of the measured field at the photodiode may depend on input amplitudes at more than two frequencies. In other words, one squeezing process may correlate amplitudes at say $f_{1}=10^{15} \mathrm{~Hz}+50 \mathrm{MHz}$ and $f_{2}=10^{15} \mathrm{~Hz}+30 \mathrm{MHz}$; another squeezing process then correlates amplitudes at $f_{2}$ and $f_{3}=10^{15} \mathrm{~Hz}-30 \mathrm{MHz}$. The output field at $f_{2}$ depends on input frequencies $f_{1}, f_{2}$, and $f_{3}$. Multiple correlations are prevented by demanding that the frequency difference of correlated amplitudes is sufficiently small so that different correlations occur at well separated parts of the spectrum. If that condition is fulfilled but "offcentered" squeezing is still allowed, then one has to take greater care when calculating the transfer functions $t_{i j}$. What if the squeezing reference frequency happens to be at $f_{0}+F$ $+5 B$, which is close enough to the measured frequency range $\left[f_{0}-B, f_{0}+B\right]$ to exhibit some influence on its vacuum noise amplitudes? The answer is derived from Eq. (22). Let us assume that the squeezing factor and phase are constant over $\left[f_{0}-B, f_{0}+B\right]$. Then, provided that $B \ll f_{0}$, the photocurrent spectral density, Eq. (6), is modified according to

$$
S_{I}^{\mathrm{QM}}(F)=P_{0} h f_{0}\left[\cosh ^{2}(r)+\sinh ^{2}(r)\right]=P_{0} h f_{0} \cosh (2 r),
$$

which means the quantum noise spectral density is necessarily increased! Therefore, any detector whose sensitivity is limited by optical quantum noise should avoid off-centered squeezing.

Again, the previous analysis is not restricted to quantumnoise spectra. The results are valid for any classical, technical noise, which is coherent or exhibits amplitude-phase correlations, which can be described by transformations such as
Eq. (26). One may say that these transformations embody the simplest kind of amplitude-phase correlations.

\section{EXEMPLARY CALCULATION}

Concluding the paper with an explicit application of our results should be helpful. We consider the following case: four classical components, one demodulation, and squeezing centered at one of the components $F_{n d}$ of the frequency matrix $\mathcal{F}$. The $N=4$ classical components have frequencies $F_{0}$ $=0 \mathrm{~Hz}, F_{1}=100 \mathrm{MHz}, F_{2}=130 \mathrm{MHz}$, and $F_{3}=230 \mathrm{MHz}$. These sideband frequencies are defined with respect to a large optical frequency $f_{0} \sim 10^{15} \mathrm{~Hz}$. The photocurrent is demodulated with $D_{0}=15 \mathrm{MHz}$. These parameters determine the frequency matrix

$$
\mathcal{F}(4,2)=\left(\begin{array}{cc}
-15 \mathrm{MHz} & 15 \mathrm{MHz} \\
85 \mathrm{MHz} & 115 \mathrm{MHz} \\
115 \mathrm{MHz} & 145 \mathrm{MHz} \\
215 \mathrm{MHz} & 245 \mathrm{MHz}
\end{array}\right) .
$$

We assume squeezing centered around frequency $F_{11}$ $=F_{20}=115 \mathrm{MHz}$, which extends locally over frequencies comparable to the detection bandwidth $B=1000 \mathrm{~Hz}$; all other frequencies contribute coherent vacuum noise. We already stated that two components of the matrix $\mathcal{F}$ are equal and the respective frequency value coincides with the squeezing reference frequency. Let us first evaluate the contributions from all unique frequencies. The corresponding vacuum fields are coherent and so the spectral density Eq. (28) simplifies to

$$
S_{n d}^{\mathrm{coh}}=h\left(f_{0}+F_{n d}\right)\left|c_{n}\right|^{2}=h\left(f_{0}+F_{n d}\right) P_{n} .
$$

These spectral densities have to be inserted into Eq. (31) and summed up for all unique frequencies

$$
\begin{aligned}
S_{I}^{\mathrm{QM}}(F)= & \frac{h}{4}\left[\left(2 f_{0}+F_{00}+F_{01}\right) P_{0}+\left(f_{0}+F_{10}\right) P_{1}+\left(f_{0}+F_{21}\right) P_{2}\right. \\
& \left.+\left(2 f_{0}+F_{30}+F_{31}\right) P_{3}\right] \approx \frac{h f_{0}}{4}\left(2 P_{0}+P_{1}+P_{2}+2 P_{3}\right) .
\end{aligned}
$$

The remaining problem is to calculate the correlated spectral density at frequency $F_{11}=F_{20}=115 \mathrm{MHz}$. For simplicity we assume that the nonlinear transfer is pure squeezing 


$$
\begin{gathered}
t_{00}\left(f_{0}+F+F_{11}\right)=\cosh (r), \\
t_{01}\left(f_{0}-F+F_{11}\right)=\sinh (r) e^{i 2 \phi}, \\
t_{10}\left(f_{0}+F+F_{11}\right)=\sinh (r) e^{-i 2 \phi}, \\
t_{11}\left(f_{0}-F+F_{11}\right)=\cosh (r) .
\end{gathered}
$$

Next, we expand the sums in Eq. (30) and substitute all known parameter values

$$
\begin{aligned}
S_{I}^{\mathrm{QM}}(F,\{(1,1),(2,0)\})= & \frac{h f_{0}}{8}\left[\mid\left(c_{1}^{*} e^{i \phi_{0}}+c_{2}^{*} e^{-i \phi_{0}}\right) \cosh (r)\right. \\
& +\left.\left(c_{1} e^{-i \phi_{0}}+c_{2} e^{i \phi_{0}}\right) \sinh (r) e^{-2 i \phi}\right|^{2} \\
& +\mid\left(c_{1}^{*} e^{i \phi_{0}}+c_{2}^{*} e^{-i \phi_{0}}\right) \sinh (r) e^{2 i \phi} \\
& \left.+\left.\left(c_{1} e^{-i \phi_{0}}+c_{2} e^{i \phi_{0}}\right) \cosh (r)\right|^{2}\right]
\end{aligned}
$$

All vacuum energies are approximated by $h f_{0}$ and $\phi_{0}$ denotes the demodulation phase. There are at least two experimentally adjustable phases: the demodulation phase $\phi_{0}$ and the squeezing phase $\phi$. What is the minimum of the spectral density depending on these two phases? Defining $\alpha$ $\equiv \arg \left(c_{1} e^{-i \phi_{0}}+c_{2} e^{i \phi_{0}}\right)$, Eq. (37) assumes the form

$$
\begin{aligned}
S_{I}^{\mathrm{QM}}(F,\{(1,1),(2,0)\})= & \frac{h f_{0}}{4}\left|c_{1}^{*} e^{i \phi_{0}}+c_{2}^{*} e^{-i \phi_{0}}\right|^{2} \times \mid \cosh (r) \\
& +\left.\sinh (r) e^{2 i(\phi-\alpha)}\right|^{2} .
\end{aligned}
$$

Minimization with respect to the squeezing phase is trivial. Setting $\phi^{\mathrm{opt}}=\pi / 2+\alpha$ and further defining $\Delta \alpha \equiv \arg \left(c_{2}\right)$ $-\arg \left(c_{1}\right)$, the minimized spectral density contribution from the squeezed part of the spectrum simplifies to

$$
\begin{aligned}
S_{\mathrm{I}}^{\mathrm{QM}}(F,\{(1,1),(2,0)\})= & \frac{h f_{0}}{4}\left[P_{1}+P_{2}+2 \sqrt{P_{1} P_{2}} \cos \left(2 \phi_{0}\right.\right. \\
& +\Delta \alpha)] e^{-2 r} .
\end{aligned}
$$

Finally, we add this result to Eq. (35), which yields

$$
\begin{aligned}
S_{I}^{\mathrm{QM}}= & \frac{h f_{0}}{2}\left[P_{0}+\frac{1}{2}\left(1+e^{-2 r}\right) P_{1}+\frac{1}{2}\left(1+e^{-2 r}\right) P_{2}\right. \\
& \left.+\sqrt{P_{1} P_{2}} \cos \left(2 \phi_{0}+\Delta \alpha\right) e^{-2 r}+P_{3}\right] \\
= & \frac{h f_{0}}{2}\left[P_{0}+\frac{1}{2}\left(1+e^{-2 r}\right) P_{1}+\frac{1}{2}\left(1+e^{-2 r}\right) P_{2}-\sqrt{P_{1} P_{2}} e^{-2 r}\right. \\
& \left.+P_{3}\right] .
\end{aligned}
$$

In the last step, we have minimized the noise power by inserting the optimal demodulation frequency $\phi_{0}^{\mathrm{opt}}=(\pi$
$-\Delta \alpha) / 2$. The reader who is exclusively interested in minimized spectral densities may easily generalize this final result including squeezing at different frequencies and an arbitrary number of classical components. The optimization procedure partly relies on the fact that the squeezing factor and phase are frequency independent. In general, experimental realization of the smallest possible noise spectral density requires further degrees of freedom, which are incorporated into the transfer functions $t_{i j}$, i.e., the light has to be filtered before the power measurement $[1,11]$. One should keep in mind that, per se, a minimal noise spectral density does not have to be optimal in terms of detector sensitivity. The reason is that noise minimization simultaneously affects the measured power of the signal. Sensitivity optimization severely depends on the detector topology [11]. It was shown in [4] that the sensitivity optimizing demodulation frequency is $\phi_{0}^{\text {opt }}=-\Delta \alpha / 2$, which goes along with a maximized noise power contribution from Eq. (39).

\section{CONCLUSION}

We have presented explicit formulas, which govern the power spectral density of photocurrents generated by power measurements of coherent and squeezed fields. We have also furnished an appropriate algorithm, which can be easily implemented in quantum-noise simulations. The algorithm is based on a few limitations concerning the classical spectrum and the squeezed spectrum of the field. However, these limitations are modest and probably insignificant for most experiments. Our results provide a long-sought-for extension of the Schottky formula $[12,13]$ to squeezed photon statistics with multiple classical components. Of special importance is that the shot noise spectrum may be calculated for any of the currently operating and next generation interferometric gravitational wave detectors. Theoretically, the method can be generalized in two ways, which we consider to be algorithmically tractable in principle. First, the transfer functions between different frequencies-which correspond to classical nonlinearities - may couple more than three amplitudes at different frequencies. Thereby, multiple squeezing centered around different frequencies with overlapping ranges of nonvanishing squeezing factor could be described. Second, one may want to give up the overlap condition between different ranges of detected field amplitudes. In that case, correlated contributions to the final current spectral density have to be revealed by means of a more elaborate scheme, which calculates the intersection boundaries of a partial overlap.

\section{ACKNOWLEDGMENTS}

We thank all members of the GEO 600 Simulation Group [14] for helpful discussions. A.F. would like to thank PPARC for financial support of this work. We also thank the SFB 407 for support. 
[1] H. J. Kimble, Y. Levin, A. B. Matsko, K. S. Thorne, and S. P. Vyatchanin, Phys. Rev. D 65, 022002 (2001).

[2] A. Buonanno and Y. Chen, Phys. Rev. D 64, 042006 (2001).

[3] J. Harms, R. Schnabel, and K. Danzmann, Phys. Rev. D 70, 102001 (2004).

[4] A. Buonanno, Y. Chen, and N. Mavalvala, Phys. Rev. D 67, 122005 (2003).

[5] S. Hild for the LSC, Class. Class. Quantum Grav. 23, S643 (2006).

[6] S. Hild, H. Grote, M. Hewitson, H. Lýck, J. R. Smith, K. A. Strain, B. Willke, and K. Danzmann, Class. Quantum Grav. 24, 1513 (2007).

[7] M. Rakhmanov, Appl. Opt. 40, 6596 (2001).
[8] C. M. Caves and B. L. Schumaker, Phys. Rev. A 31, 3068 (1985).

[9] H. Vahlbruch, S. Chelkowski, B. Hage, A. Franzen, K. Danzmann, and R. Schnabel, Phys. Rev. Lett. 97, 011101 (2006).

[10] A. Buonanno and Y. Chen, Phys. Rev. D 65, 042001 (2002).

[11] J. Harms, Y. Chen, S. Chelkowski, A. Franzen, H. Vahlbruch, K. Danzmann, and R. Schnabel, Phys. Rev. D 68, 042001 (2003).

[12] W. Schottky, Ann. Phys. 57, 541 (1918).

[13] J. D. Spear, Rev. Sci. Instrum. 76, 076101 (2005).

[14] http://www.sr.bham.ac.uk/dokuwiki/doku.php?id = geosim:home 